\title{
El niño y el sistema de numeración decimal
}

\author{
Evelio Bedoya y Mariela Orozco
}

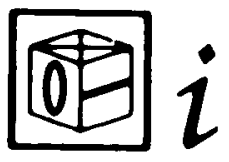

Se plantean en este artículo las dificultades que pueden experimentar los niños para apropiarse de las nociones implícitas en el sistema de numeración decimal, tales como las unidades compuestas o el valor posicional, y que llevan implícitas otra serie de nociones que, si no se explicitan por el maestro en su didáctica, impedirán la correcta apropiación de la totalidad del sistema.

\section{LA DECENA}

Aunque los diccionarios definen la decena como un «conjunto de diez unidades», realmente, de acuerda con sus usos, constituye una unidad cuyo valor, relativo a los objetos discretos y análogos que la configuran, es diez. Diez objetos cualesquiera y aislados no constituyen por sí solos una decena, a no ser que en la mente de un sujeto se configure con ellos una unidad a partir de algunas relaciones y operaciones complejas. Una decena es una unidad de un orden superior al de las unidades que la conforman. Es una unidad de unidades.

\section{EL NUMERO DIEZ: LA DECENA Y LA CENTENA}

El número diez, que no el numeral, en tanto clase conceptual, es un concepto más general que la decena; ésta sólo es un ejemplo de dicha clase, como lo son un grupo de diez personas o de diez objetos cualesquiera. A su vez, el número diez es un representante de la clase conceptual mucho más general que es el concepto de número natural. Asimismo, una centena es un doble ejemplo del concepto de número diez. En una centena hay diez decenas; una centena es, por tanto, una unidad de unidades compuestas (decenas), las cuales son, a su vez, unidades compuestas de unidades simples. Si llamamos a la centena unidad de orden 2, a la decena unidad de orden uno y a la unidad simple, unidad de orden cero, podemos decir que una centena es una unidad compuesta de unidades de orden uno, que a su vez son unidades compuestas de unidades de orden cero. 
Formalmente, la unidad decimal o unidad en base 10 se define como la clase conceptual cuyos componentes son las unidades decimales de órdenes 0,1 , 2,3 , etc.:

Unidad decimal de orden 0 Unidad decimal de orden 1 Unidad decimal de orden 2 Unidad decimal de orden $n$

1
10
100
$100 . . .0$

$\mathrm{n}$ ceros

\section{EL SISTEMA DE NUMERACION DECIMAL (S.N.D.)}

En general, un sistema de numeración está constituido por un conjunto de números, una colección de símbolos y signos básicos y unas reglas que permiten expresar o representar los números del conjunto. El conjunto de números, al cual nos referimos en este artículo, es el de los naturales y el sistema de numeración es el decimal. Los signos o símbolos básicos del S.N.D. son: 1, 2, 3, 4, $5,6,7,8,9,0$, y un punto (.) para indicar unidades de mil, de millón, etc.

Para representar un número natural, en el S.N.D., se deben seguir las siguientes reglas: solamente se escriben las cifras que especifican el número de unidades que lo componen. Estas cifras se escriben, una a continuación de la otra, de izquierda a derecha, en relación decreciente con respecto al orden de las unidades. El nombre del número se forma expresando el número de unidades de cada orden que contiene.

Estas reglas dotan a cada una de las cifras de un doble valor: el valor correspondiente al número de unidades y el valor relativo al orden. Este último se infiere de la posición que la cifra ocupa en el numeral. La técnica conocida como «valor de posición», que la escuela tradicional suele implementar para manejar los números naturales en el S.N.D., constituye una simplificación exagerada de las anteriores reglas.

Desde una perspectiva matemática y cognitiva, para manejar el S.N.D., esto es, para manejar los números naturales en dicho sistema, es necesario construirlos progresivamente en los diferentes períodos que configuran el sistema. Un período está definido por los números comprendidos entre dos unidades decimales de órdenes consecutivos, incluyendo la unidad de orden inferior. El primer período está constituido por los números naturales entre 0 y 10 ; el segundo, por los números naturales entre 9 y 100; el tercero, por los números naturales entre 99 y 1.000 y así sucesivamente.

Construir un número natural, en un período determinado, exige manejar:

- números y unidades de períodos y órdenes anteriores al período u orden correspondiente al número dado;

- algunos términos de la sucesión de unidades

$$
\begin{array}{llll}
1 \times 10^{1}, & 2 \times 10^{1}, & \ldots, & 9 \times 10^{1}, \\
1 \times 10^{2}, & 2 \times 10^{2}, & \ldots, & 9 \times 10^{2}, \\
1 \times 10^{3}, & 2 \times 10^{3}, & \ldots, & 9 \times 10^{3}, \text { etc.; o en forma más abreviada: } \\
1 \times 10^{n}, & 2 \times 10^{n}, & \ldots, & 9 \times 10^{n},
\end{array}
$$

- La secuencia de unidades $1,2,3,4 \ldots, 9, \mathrm{y}$

- La operación $\ll n+1 »$. 
Para construir una unidad decimal y en general un número, en un cierto período, es necesario recurrir aditiva o multiplicativamente a unidades y números de períodos anteriores. Por ejemplo, definir la unidad de orden $3\left(10^{3}\right)$ exige recurrir aditiva o multiplicativamente a las unidades de órdenes $2\left(10^{2}\right), 1(10)$ у 0 (1):

$$
\begin{aligned}
& 10^{3}=\underbrace{10^{2}+10^{2}+\ldots+10^{2}}_{10 \text { veces } 10^{2}}=10 \times 10^{2} \\
& =\underbrace{(10+10+\ldots+10)}_{10 \text { veces } 10}+\underbrace{(10+10+\ldots+10)}_{10 \text { veces } 10}+\ldots+\underbrace{(10+10+\ldots+10)}_{10 \text { veces } 10}
\end{aligned}
$$

10 veces $\underbrace{(10+10+\ldots+10)}_{10 \text { veces } 10}$

$=10 \times(10 \times 10) \quad[10$ veces $(10$ veces 10$)]$

Análogamente, se puede demostrar que la unidad de orden $3\left(10^{3}\right)$ es 100 veces 10 y 1.000 veces 1 . En otras palabras, la unidad de orden 3 es una unidad compuesta de unidades de orden 2 , las cuales a su vez son unidades compuestas de unidades de orden 0 . El número o numeral 243 (en el tercer período), contiene las unidades de órdenes 2,1 y 0 y números o numerales de los períodos tercero, segundo y primero. Veamos:

$$
\begin{array}{r}
243=200+40+3=240+3=200+43 \\
=2 \times 10^{2}+4 \times 10+3=24 \times 10+3=2 \times 10^{2}+43
\end{array}
$$

Igualmente, para la construcción de un número natural cualquiera o de una unidad decimal, es necesario manejar la sucesión de unidades $1 \times 10^{n}, 2 \times 10^{n}$, $3 \times 10^{n}, \ldots, 9 \times 10^{n},(n=1,2,3$, etc.). Examinemos el siguiente par de ejemplos:

a) $52=50+2=5 \times 10+2=(40+10)+2=(49+1)+2$

b) $100=90+10=9 \times 10+10=99+1$

De cierta manera, 100 también es el resultado de la enumeración secuencial de los diez primeros términos de la sucesión de unidades: 10, 20, 30, ., 90, 100.

Formalmente se puede afirmar que la construcción del concepto de número natural exige, igualmente, extrapolar de manera sucesiva la secuencia de dígitos: $1,2,3,4,5,6,7,8,9$. Esta extrapolación, conjuntamente con el manejo de la sucesión de unidades $1 \times 10^{n}, 2 \times 10^{n}, \ldots, 9 \times 10^{n}$, lo llamamos proceso de recurrencia de la sucesión de unidades. La construcción del concepto de número natural como sucesión numérica exige la extrapolación sucesiva de los dígitos y, de alguna manera, el proceso de recurrencia de la sucesión de unidades.

$$
\begin{aligned}
& 1,2,3,4,5,6,7,8,9,10 \text {; } \\
& 11,12,13,14,15,16,17,18,19,20 \text {; } \\
& 21,22,23,24,25,26,27,28,29,30 \text {; } \\
& 31,32,33,34,35,36,37,38,39,40 \text {; y así sucesivamente. }
\end{aligned}
$$

La operación $\ll n+1 »$, además de permitir construir inductivamente la sucesión de números naturales, posibilita, mediante la iteración aditiva del 1, establecer las relaciones «el siguiente de» y «el anterior de» entre dos números 
consecutivos. A partir de estas relaciones se pueden definir las relaciones de orden «menor que» $\mathrm{y}$ «mayor que» entre números naturales. Por ejemplo, 8 es menor que $10(8<10)$ porque $1 \varnothing=8+2=(8+1)+1=9+1$.

\section{RELACIONES Y OPERACIONES NECESARIAS EN EL NIÑO PARA CONSTRUIR EL SISTEMA}

Las características del sistema exigen a los niños construir progresivamente unidades compuestas partiendo de unidades de órdenes inferiores, elaborando, de esta manera, una secuencia de unidades de orden $0,1,2,3$, etc.; igualmente, extrapolar, de manera sucesiva, los dígitos entre los términos de la sucesión de unidades. Para lograr esta construcción, es preciso que el niño realice operaciones de composición y descomposición y establezca relaciones de inclusión, equivalencia y recurrencia.

Steefe (1990) señala que el niño necesita realizar composiciones y descomposiciones de unidades para asignar significado a un número como el 20 . Kamii (1986) y Gómez-Granell (1987) plantean, que la construcción de la noción de valor de posición involucra la operación de multiplicación. Kamii (1986) además señala que, para comprender el valor de posición, el niño igualmente necesita manejar la relación de parte a todo. El manejo de esta relación, por supuesto, involucra trabajar simultáneamente relaciones de equivalencia y de inclusión.

Señalar que el niño necesita efectuar operaciones de composición y descomposición y establecer relaciones de equivalencia, no quiere decir que la maestra debe ponerle tareas sobre estas operaciones y relaciones para que él aprenda a manejarlas. Se trata de operaciones y relaciones mentales que el niño debe poner en funcionamiento para resolver, comprensiva y adecuadamente, tareas sobre el S.N.D.

Reconocer y manejar el carácter del S.N.D. permite a la maestra proponer tareas que resulten adecuadas a las características del sistema y que susciten la reflexión del niño; igualmente, posibilita el manejo de un marco conceptual para analizar las producciones de los niños al resolverlas.

Para ejemplificar este supuesto pedagógico, utilizaremos el modelo del ábaco. Suponemos que este modelo resulta una manera muy sencilla y menos usual de ejemplificar el problema de la representación de numerales y que podría ayudar a descentrar a la maestra de las rutinas que generan las conversiones entre las unidades que componen el sistema. Nos referimos a un ábaco para niños, con 10 hiladas y 10 cuentas en cada hilada. En estos ábacos, las hiladas representan a las unidades de órdenes 0 a 9 y cada hilada corresponde a un período. Las cuentas en cada hilada representan los correspondiente términos en la sucesión de unidades, y las 10 cuentas, el número de unidades en el respectivo período.

La maestra pide a Juanito que represente el 222 en un ábaco. Juanito ha trabajado la construcción del sistema utilizando tareas como las de los collares (que presentaremos posteriormente) y trabajando con los cubos de Dienes (1965). Respondiendo a la solicitud de su maestra, el niño mueve hacia la izquierda dos cuentas, separándolas de las demás, en las tres primeras hiladas, conformando, en el ábaco, la siguiente configuración (ver Figura $n .^{\circ} 1$ ).

Hiladas con bolitas de colores ensartadas y en algunas de las hiladas, unas bolitas distantes de las restantes, es la configuración que el niño, o cualquier 


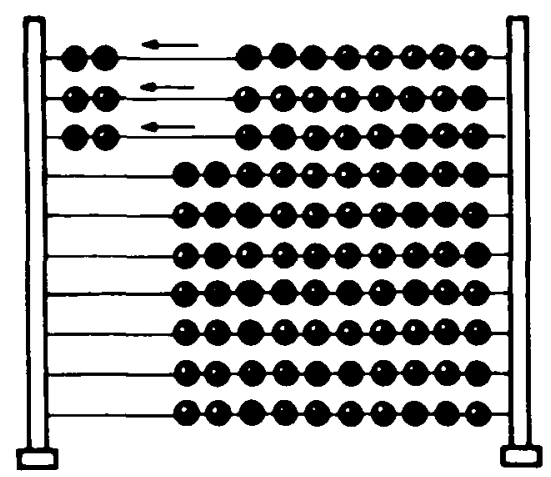

sujeto, tiene frente a sí. Para comprender que esta configuración representa el 222, el niño tiene que entender que las dos cuentas, en cada una de las hiladas, tienen un valor diferente. En la primera, las dos cuentas representan dos unidades de uno; en la segunda, dos unidades que valen diez y en la tercera, dos unidades que valen cien.

¿Cómo lee o significa la maestra lo que el niño hizo? Si Juanito entiende que las dos cuentas en la primera hilada valen dos, ella puede interpretar que el niño maneja unidades de orden 0 y la secuencia numérica verbal, hasta ese número. Esta es una comprensión que los niños desarrollan muy temprano y que Steefe (1990) denomina manejo de la secuencia numérica inicial.

Como la producción de Juanito en el ábaco igualmente revela a la maestra que él representa el «20» y el «200» (con las cuentas que separa en la segunda y tercera hiladas), ella le pregunta: ¿Qué quieren decir estas dos cuentas aquí y aquí? señalando las cuentas en la segunda y la tercera hiladas. El niño responde: «Estas son dos de diez y estas dos de cien.» ¿Y cuántas de diez hay en ésta?, pregunta la maestra, señalando la tercera hilada. El niño contesta: «Diez de diez.»

Que las 2 cuentas de la segunda hilada sean para Juanito «dos de diez» y las de la tercera, «dos de cien», permite a la maestra inferir que el niño efectivamente ha representado unidades de orden 1 y 2 . La expresión «dos de diez» igualmente le revela que el niño comprende que una cuenta equivale a 10 cuentas de la primera hilada y que 2 cuentas equivalen a 20. La respuesta de Juanito «dos de cien», revela que él establece la equivalencia entre una unidad de orden 2 y las de orden 0 . Para poder establecer estas equivalencias el niño debe descomponer las unidades de orden superior en las de orden inferior.

Sin embargo, la expresión «diez de diez» es la respuesta que efectivamente la convence, porque le revela que el niño simultáneamente ha realizado una composición multiplicativa y ha establecido una relación de equivalencia entre la unidad de segundo orden y las de primer orden y, por supuesto, para establecer la equivalencia, debe descomponer las unidades de orden superior en las de orden inferior. 
Para entender el valor de las cuentas en la segunda hilada, los niños tienen que ser capaces de componer 10 objetos en una totalidad y manejar el 10 como una unidad, o sea, elaborar lo que Steffe (1990) ha llamado una unidad «compuesta» o «unidad de unidades»; en nuestro lenguaje, una unidad de orden 1 que incluye las unidades de orden 0 . Para entender el valor de cada cuenta de la tercera hilada, los niños deben ser capaces de componer 100 unidades de orden 0 ó 10 unidades de orden 1 en una unidad de orden 2 y manejar el 100 como una unidad de unidades compuestas. De esta manera, para asignar a las cuentas el valor correspondiente a una unidad de segundo orden, deben componer 10 unidades de primer orden en una unidad de segundo orden, o unidad compuesta de unidades compuestas; igualmente, deben descomponer la unidad de segundo orden en las unidades de órdenes 1 y 0 y establecer equivalencias entre ellas.

Sin embargo, nos faltaría analizar cómo realizan los niños la extrapolación sucesiva de los dígitos. Para esto examinaremos el trabajo de una maestra que ha pedido a los niños que hagan collares, diademas y pulseras utilizando cuerdas y macarrones. Cada adorno debe tener 10 macarrones. Cada vez que los niños terminan uno, la màestra les pregunta: ¿Cuántos collares tienes?, ¿cuántos macarrones tiene tu collar (pulsera o diadema)?, ¿cuántos collares (pulseras o diademas) tienes? Una vez que los niños han terminado de hacer varios collares (pulseras y diademas), la maestra les pregunta: si juntamos los collares, pulseras y diademas, ¿cuántos macarrones tienes?

Examinemos la respuesta de Alejandra ante esta última pregunta. Para establecer cuántos macarrones tiene én 2 collares, 2 pulseras y 2 diademas, que ha construido, Alejandra dice: «Diez», haciendo a un lado el primer collar; luego, toca uno a uno los macarrones del segundo y dice: «11, 12, 13, 14, 15, $16,17,18,19,20$ "; entonces, coge una pulsera y dice veintiuno, veintidós, veintitrés, ..., veintinueve, veintidiez, mientras toca uno a uno los macarrones. «Treinta», la corrige la maestra. La niña continúa con la otra pulsera. «Treinta y uno, treinta y dos, treinta y tres, ..., treinta y nueve», y voltea a mirar a la maestra con un gesto de interrogación en su cara. La maestra le dice: «cuarenta». Alejandra continúa enumerando de la misma manera los macarrones en las diademas hasta sesenta, cuando termina de contar los macarrones en los collares, pulseras y diademas que ha hecho. La maestra le pregunta: «¿Cuántos collares, pulseral y diademas tienes en total?» La niña los toca uno a uno diciendo: $\ll 1,2,3,4,5,6,7 »$.

Alejandra retira el primer collar y ha dicho «diez», sin volver a contar los macarrones. Esta manera de manejar el collar y de asignarle un valor permite a la maestra inferir que Alejandra comprende que en cada collar hay 10 y que maneja el 10 como unidad de unidades. Cuando llega al treinta, la niña dice: «veintidiez». Como la maestra la corrige, cuando llega al «treinta y diez» no lo dice, sino que mira a la maestra, como solicitándole que le diga el nombre del nuevo número, así cuenta hasta sesenta. No existen registros previos que muestren que Alejandra enumera hasta este número. Suponemos que ella puede hacerlo porque cae en cuenta de otra de las regularidades del sistema y extrapola la secuencia de dígitos y la maneja en la secuencia de términos de la sucesión que empieza a construir, cuyo nombre aún no sabe. Finalmente, la niña no sabe cuántos dieces ha contado. Ella solamente enumera y construye la secuencia numérica hasta sesenta, utilizando material concreto que sirve para representar la unidad de diez. 


\section{CONCLUSIONES}

El análisis realizado nos obliga a reconocer la dificultad que encierra el manejo del sistema. Desafortunadamente la escuela la desconoce y reemplaza esta complejidad por un manejo burdo del «valor de posición», causando verdaderos desastres en la comprensión que el niño desarrolla sobre el número y el S.N.D.

Sería necesario resaltar, una vez más, de qué manera la maestra utiliza sus conocimientos sobre el sistema. En primer lugar, ella puede escoger tareas que resultan adecuadas y formular preguntas que permitan al niño reflexionar sobre el sistema. En segundo lugar, la maestra maneja un marco conceptual que le permite interpretar la producción de los niños, entendiendo su manera de hacer matemáticas.

\section{Referencias}

Bassedas, M.; Sellarés, R. (1986). La Evolución de la Comprensión de las Operaciones Aritméticas Elementales. En: IMIPAE, La Pedagogia Operatoria Hoy. Barcelona: Ajuntament de Barcelona, pp. 81-101.

Dienes, Z. P. (1965). La Matemática Moderna en la Enseñanza Primaria. Barcelona: Teide. 7. ${ }^{\text {a }}$ Edición, 1981

Gómez-Granell G., (1986). La Función del Dibujo en la Construcción de los Formalismos Matemáticos. Cuadernos de Psicologia, Vol 8, N. ${ }^{\circ}$, pp. 155-178.

Kami, C., (1985). El Niño Reinventa la Aritmética. Implicaciones de la Teoría de Piaget. Madrid: Visor

Kamn, C., (1986). Place Value: An Explanation of Its Difficulty and Educational Implications for the Primary Grades. Journal of Research in Childhood Education. Abril (Versión en Castellano: Valor de Posición: Una Explicación de sus Dificultades e Implicaciones Educacionales pa. ra los Alumnos de Primaria. Cuadernos de Psicología, Vol. 9, N.², 1988, pp. 112-135).

Smith, D. E.; Ginsburg, J. (1979). De los Números a los Numerales y de los Numerales al Cálculo. En Newman, J. R., El Mundo de las Matemáticas, Barcelona: Grijalbo.

Stefre, L., (1990). Children's Construction of Meaning for Arithmetical Words. A Curriculum Problem. (Versión en Castellano: Cómo construye el Niño la Significación de los Términos Aritméticos. Un Problema Curricular. Cuademos de Psicología, Vol. 11, N. 1 y 2 . 1990). 


\section{El niño y el sistema de numeración decimal. Evelio Bedoya y Mariela Orozco. \\ CL\&E, 1991, 11-12, pp. 55-62}

Resumen: El articulo intenta presentar algunas elaboraciones que podrian justificar las dificultades que los niños tienen para manejar el sistema de numeración decimal y ejemplificar la comprensión que la maestra debe poseer para trabajarlo. Para esto presentamos un descripción del sistema y las operaciones y relaciones que los niños requieren para manejarlo, analizando producciones de niños al resolver tareas que se adaptan a las características del sistema.

Datos sobre los autores: Evelio Bedoya es profesor del Departamento de Matemáticas y Mariela Orozco del Departamento de Psicología, de la Universidad del Valle.

Dirección: Universidad del Valle. Departamentos de Matemáticas y de Psicología. Cali. Colombia.

Agradecimientos: Este artículo es producto de las discusiones sostenidas en el Seminario Permanente de Investigaciones en la Enseñanza de la Matemática que el Programa «El Niño y la Escuela» impulsa desde el Departamento de Psicología de la Universidad del Valle, Cali, Colombia.

(C) De todos los artículos. Deberá solicitarse por escrito autorización de CL\&E y de los autores para el uso en forma de facsímil, fotocopia o cualquier otro medio de reproducción impresa. CL\&E se reserva el derecho de interponer las acciones legales necesarias en aquellos casos en que se contravenga la ley de derechos de autor. 